\title{
Study of Degradation of Polystyrene, Using Ultraviolet Spectrophotometry
}

\author{
Mary Jane Reiney, Max Tryon, and B. G. Achhammer
}

\begin{abstract}
The role of ultraviolet radiant energy and effect of presence of monomer on degradation of polystyrene were studied by means of ultaviolet absorption techniques. It was found that exposure of pure polystyrene to ultraviolet radiant energy resulted in increased absorption in the ultraviolet region of 280 to 400 millimicrons, which advanced progressively as exposure time increased. The presence of monomer increased the rate of degradation without altering the general type of absorption. A post-radiation effect was noted in both the purified polymer and polymer containing monomer styrene. Again, the presence of monomer increased the rate of progress of the post-radiation effect. Possible mechanisms are postulated for the ultraviolet degradation, and the concept of entrapped free radicals is considered as a possible explanation for the post-radiation effect observed.
\end{abstract}

\section{Introduction}

It has been shown by infrared spectrophotometric measurements that carbonyl and hydroxyl groups are formed in polystyrene on exposure to ultraviolet radiant energy in the presence of oxygen [1, 2]. ${ }^{1}$ The volatile products given off and measured by mass spectrometry and the changes in solubility and oxygen content on exposure of polystyrene to heat and/or ultraviolet radiant energy in vacuum and in oxygen have also been reported [3]. This report presents the changes in the ultraviolet spectrum of polystyrene as a result of exposure to ultraviolet radiant energy in air. A mechanism is suggested to describe possible chemical changes in the polymer that could be responsible for the variation of the ultraviolet spectrum of polystyrene, both on exposure of the polymer to ultraviolet radiant energy and on subsequent storage in the absence of light.

\section{Materials and Methods}

The polystyrene samples used in this study are described in table 1. All samples were prepared by the Dow Chemical Co. Samples A and E1 are commercial resins; they do not contain any compounding ingredients and were used as received. Samples $\mathrm{W}, \mathrm{X}$, and $\mathrm{Y}$ were prepared by polymerizing purified monomer without catalyst or solvent in a nitrogen atmosphere.

TABLE 1. Description of samples of polystyrene

\begin{tabular}{|c|c|c|}
\hline Sample & $\begin{array}{l}\text { Approximate } \\
\text { molecular } \\
\text { weight }\end{array}$ & $\begin{array}{l}\text { Polymeriza- } \\
\text { tion tem- } \\
\text { perature }\end{array}$ \\
\hline $\begin{array}{l}\mathrm{A} \\
\mathrm{E} 1 \\
\mathrm{~W} \\
\mathrm{X} \\
\mathrm{Y}\end{array}$ & $\begin{array}{l}\text { a } 308,000 \\
\text { a } 299,000 \\
\text { b } 500,000 \\
\text { b } 237,000 \\
\text { b } 110,000\end{array}$ & $\begin{array}{c}{ }^{\circ} \mathrm{C} \\
60 \\
120 \\
180\end{array}$ \\
\hline
\end{tabular}

a Weight average 0 btained by light scattering.

b Number average obtained from osmotic pressure.

1 Figures in brackets indicate the literature references at the end of this paper.
The spectrophotometric measurements on these samples as received are designated by the sample letters alone. The measurements made after partial purification of the samples by a "frozen benzene technique" [2] are designated by the sample letters followed by P. Sample X was also subjected to purification by solution in methyl ethyl ketone and precipitation with isopropanol three times; the samples from the first, second, and third precipitations are designated $\mathrm{X}_{1}, \mathrm{X}_{2}$, and $\mathrm{X}$ 3, respectively.

The technique for preparation of the samples in film form of various thicknesses has been described previously [2]. Casting of the thicker films on a surface of regenerated cellulose, instead of directly on glass, facilitated their removal because wetting the opposite side of the cellulose with water swelled the cellulose and broke the bond between it and the polystyrene film.

Exposure of the films to ultraviolet radiant energy was accomplished by placing the films in holders on a revolving table 6 in. from an $\mathrm{S}-1$ sunlamp. The exposure was done in air, and the temperature at the table was $60^{\circ} \mathrm{C}$. This sunlamp equipment is described in method No. 6021 of Federal Specification L-P-406a [4].

A Beckman model DU spectrophotometer with ultraviolet accessories was used for all the transmission measurements. ${ }^{2}$ For solution work, silica cells of $1.000-\mathrm{cm}$ path length were used.

\section{Effect of Film Thickness on Transmittance}

In the same manner that the concentration of a solution for a given light path length will dictate the spectrum of polystyrene obtained, the thickness of films of the polymer will be the controlling factor in determining its absorption spectrum. The effect of film thickness on the spectrum of polystyrene films of sample XP is shown in figure 1.

Films of thickness equivalent to that of curve $\mathrm{E}$ of figure 1 were selected for the present study because they are thick enough to be mechanically stable;

2 The various spectral transmission and absorption terms used in this paper are based on the terminology recommended by Kasson S. Gibson in NBS Circular 484 , Spectrophotometry (200 to 1,000 millimierons) (Sept. 15, 1949). 


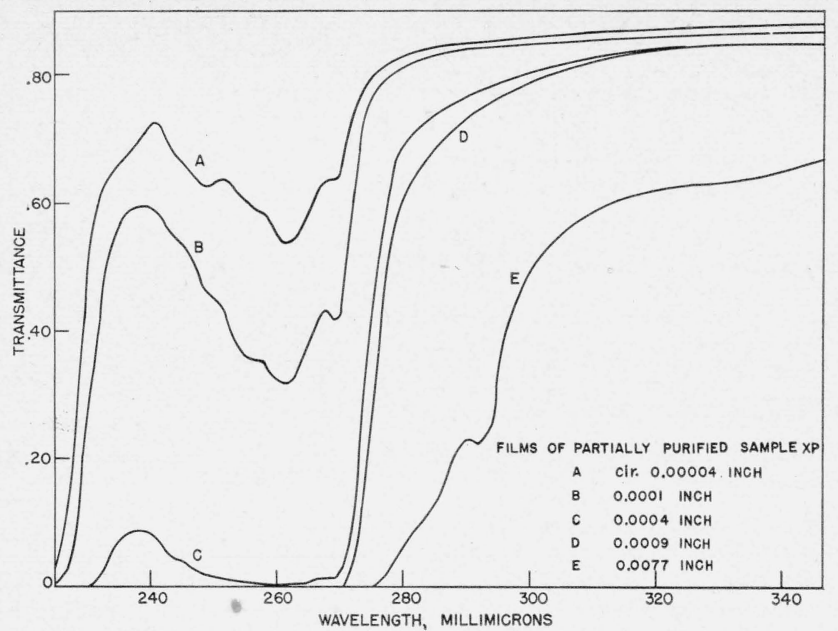

FIGURE 1. Effect of film thickness on the ultraviolet transmittance of polysiyrene.

they are not too thick to show the shape of the absorption curve in the region 275 to $350 \mathrm{~m} \mu$ where the greatest differences between the polymers studied were obtained; and their strong absorptance ${ }^{3}$ below $270 \mathrm{~m} \mu$ is relatively unimportant to this study. Smakula [5] states that the absorptance of polystyrene at about $260 \mathrm{~m} \mu$ is due to the phenyl residues. These would not be expected to change appreciably on degradation of the polymer. Furthermore, monomer styrene, which has been suggested as playing an important part in the discoloration phase of the degradation of the polymer [1], is known to absorb in the ultraviolet at $291.5 \mathrm{~m} \mu$. The fact that the absorption band at $291.5 \mathrm{~m} \mu$ is present in curve $\mathrm{E}$ indicates that the purification of the sample was not complete and that some monomer remained in the sample.

\section{Transmittance of Undegraded Polymers}

Figure 2 presents the spectra of films of the various polymer samples studied. It is thought that the differing spectra cannot be ascribed to the moderate thickness variations of the films. This is substantiated by the measurements made on solutions of 18 $\mathrm{g}$ /liter concentration of WP, XP, and YP in benzene, which are presented in figure 3 . The solution spectra of these three samples bear out the relationship shown by the spectra of these samples in film form.

Moreover, the variation in the spectra cannot be ascribed to the molecular weight differences of the samples. McGovern and coworkers [6] found that the same spectrum was obtained at wavelengths of 282 and $291 \mathrm{~m} \mu$, regardless of the molecular weight of the polystyrene. Meehan [7] showed that the specific extinction coefficient, referred to weight concentration of polystyrene, at $260 \mathrm{~m} \mu$ is independent of molecular weight. Newall [8] measured five polystyrene samples and observed that neither molecular weight nor variables of the polymerization reaction

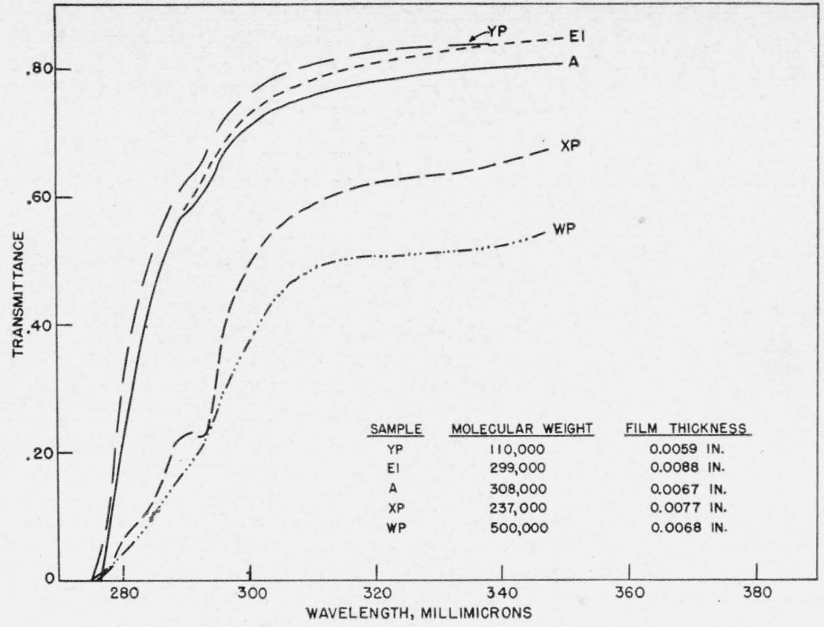

FIGURE 2. Ultraviolet transmittance of partially purified polystyrenes.

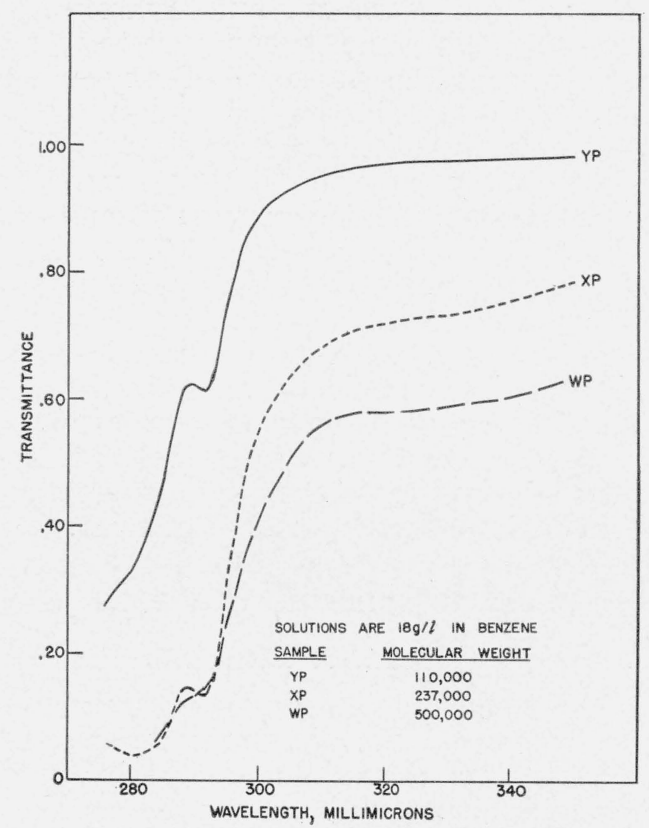

FIGURE 3.. Ultraviolet transmittance of partially purified poiystyrenes.

affected the spectrum of polystyrene at the shorter wavelengths.

The spectrum of polystyrene, however, is a function of the purity of the sample. Sample X was carefully purified by repeated solution in methyl ethyl ketone and precipitation with isopropanol. The X 3 curve in figure 4 represents the pure polymer, and the other spectra denote varying degrees of contamination retained by the polymer. The X polymer shows marked regions of absorptance not characteristic of pure polystyrene. Figure 5 combines the spectra of $18 \mathrm{~g} /$ /iter solutions of X3 and X with those of the unpurified polymers $\mathrm{W}$ and $\mathrm{Y}$. The difference in purity of the polymers $\mathrm{W}, \mathrm{X}$, and $\mathrm{Y}$ is clearly shown,

3 Absorptance is defined as " 1 -Transmittance." 


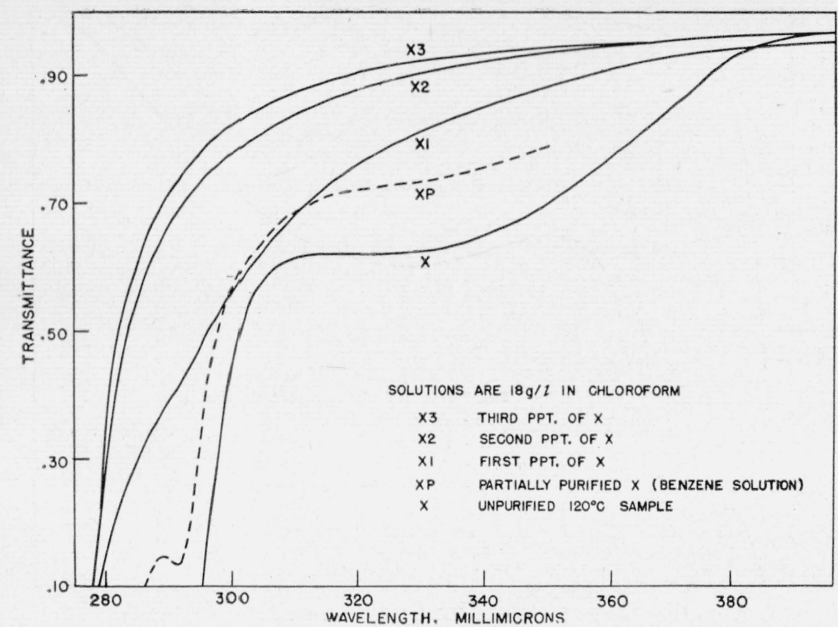

FIGURE 4. Ultraviolet transmittance of polystyrene of varying degree of purity.

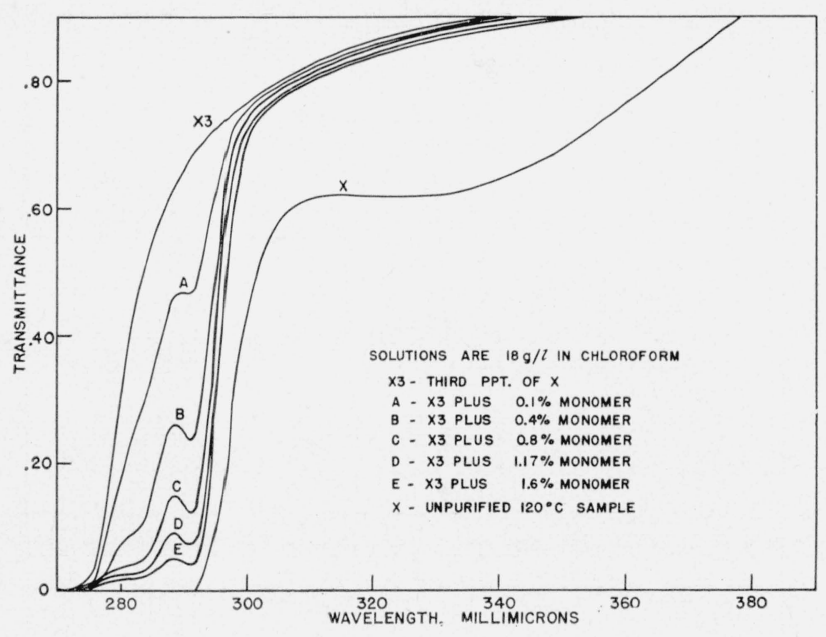

FIGURE 6. Effect of monomer and impurities on the ultraviolet transmittance of polystyrene.

particularly the considerable contamination in the $\mathrm{W}$ polymer. It is reasonable, therefore, that an identical purification procedure applied to each polymer might not produce samples of the same degree of purity, thus leading to the differing spectra exhibited by the samples WP, XP, and YP in figure 3 .

\section{Effect of Styrene Monomer on Polymer Spectra}

In order to determine the part that residual monomer contributes to the contamination of the unpurified polymer, styrene was added to solutions of pure polystyrene (precipitate X3 of sample X). Solutions containing approximately $0.1,0.4,0.8,1.17,1.6$, $2.0,2.8,5.0$, and 7.35 percent of styrene were prepared. Figure 6 shows the curve of the pure polymer, those of the first five solutions in the monomer-

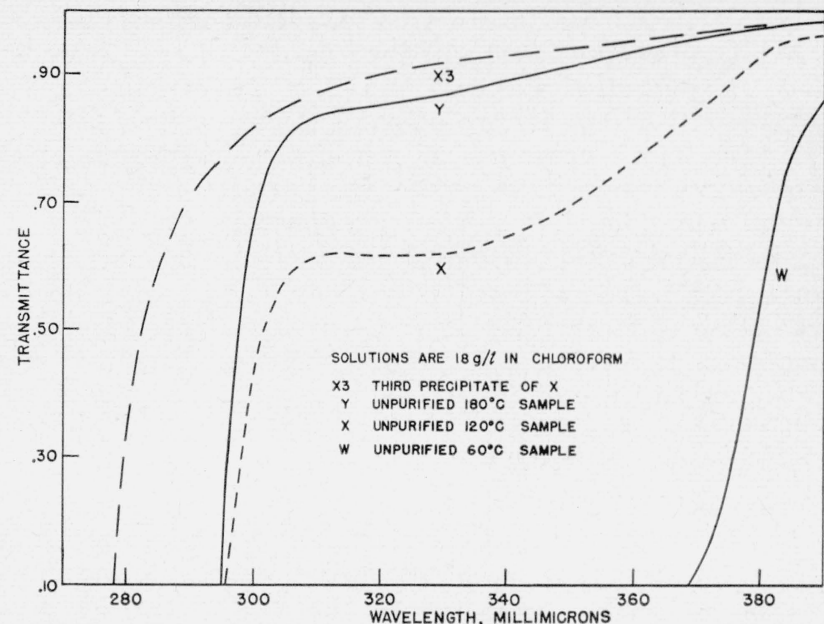

FIGURE 5. Ultraviolet transmittance of polystyrenes of varying degree of purity.

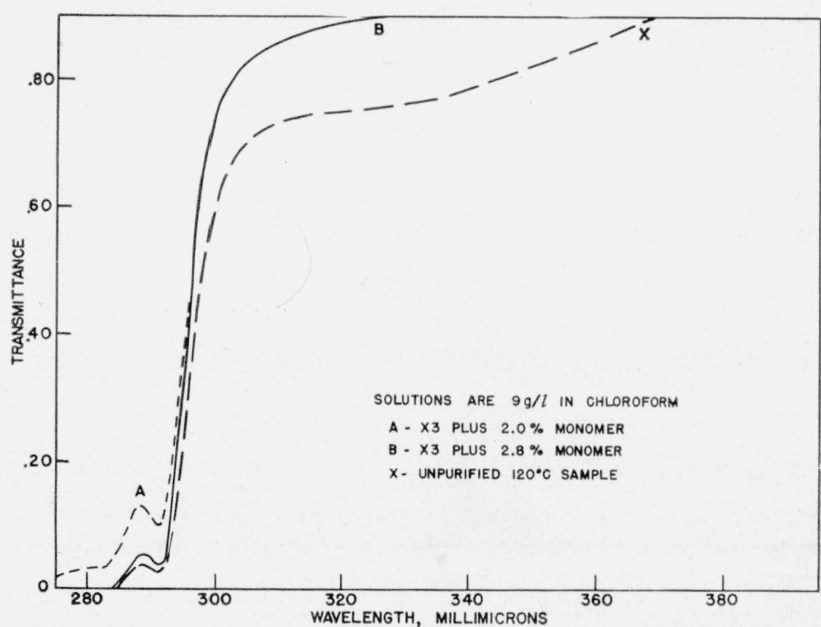

Figure 7. Effect of monomer and impurities on the ultraviolet transmittance of polystyrene.

addition series and that of the unpurified polymer, all at $18 \mathrm{~g}$ /liter concentration. The effect of increasing monomer content is clearly shown. There is also an absorption band from 310 to $385 \mathrm{~m} \mu$ in the unpurified sample. That this band cannot be attributed to monomer contamination is evident from the spectra in figure 7 made with solutions of $9 \mathrm{~g}$ /liter concentration. Even when the $292 \mathrm{~m} \mu$ monomer band of $\mathrm{X}$ is approximately duplicated by the addition of 2.8 percent of monomer to X3 there is still no indication of the 310 to $385 \mathrm{~m} \mu$ band of X present in the spectrum of the latter solution. Changes in absorptance in the 310 - to $385-\mathrm{m} \mu$ wavelength region were noted on exposure of the films to ultraviolet radiant energy, and a more detailed investigation of this region is described in section 8 .

This failure of residual monomer to account for the long-wavelength contamination of the unpurified 
polymers is further shown by examination of figure 8. The pure polymer solution containing 7.35 percent of added monomer shows a spectrum comparable to that of $\mathrm{W}$ only below approximately $295 \mathrm{~m} \mu$. The presence of monomer causes no appreciable change in the spectrum of the pure polymer at wavelengths longer than $300 \mathrm{~m} \mu$. This figure shows the two monomer absorption bands at approximately 282 and $291.5 \mathrm{~m} \mu$ more clearly than any of the preceding figures and illustrates that monomer is responsible for the decreased transmittance ${ }^{4}$ of W below $295 \mathrm{~m} \mu$.

Figure 9 presents the spectra of three solutions of styrene monomer alone in chloroform. The absorption bands at 282 and $291.5 \mathrm{~m} \mu$ are shown, as well as the intense band at $248 \mathrm{~m} \mu$; but even when the spectrum of a very concentrated solution is considered (curve C), there is no indication of an absorption band at longer wavelengths. The absorption bands shown are due to the presence of the double bond in conjugation with the benzene ring and form the very characteristic spectrum that is distinguishable from that of saturated derivatives and from that of unsaturated compounds without the double bond in conjugation with the benzene ring [9]. This pattern of absorptance displayed by the monomer permits quantitative determination of styrene in polystyrene spectrophotometrically $[6,8]$ as well as the determination of monomer styrene in ethylbenzene [10].

\section{Effect of Monomer on Polystyrene Degradation}

To study the effect of styrene monomer on the degradation of polystyrene, two films were prepared. One was cast from a benzene solution of sample X3 and the other from a benzene solution of sample X3 to which 2 percent of monomer was added. The films were made by the same procedure and measured 0.0066 and 0.0068 in. thick, respectively. They were exposed to ultraviolet radiant energy from an $\mathrm{S}-1$ sunlamp at $60^{\circ} \mathrm{C}$ in air. Ultraviolet transmission measurements of these films at intervals during their exposure give an insight into the degradation of the pure polymer and polymer contaminated with monomer but not with other impurities. Figure 10 shows the effect of exposure to $\mathrm{S}-1$ radiant energy on the transmittance of the pure film in the ultraviolet region of the spectrum, and figure 11 shows similar information for the film prepared from the solution to which monomer was added. A progressive general increase in absorptance is noted in each case but is initially more severe for the film containing monomer. The 20and $50-\mathrm{hr}$ spectra of figure 11 bear a resemblance to the partially purified XP film spectrum noted previously. Both films yellowed very slightly as they were exposed, the monomer-containing film slightly more so than the pure film.

4 "Transmittance," $T$, is defined as the ratio of the radiant energy leaving the sample to the incident radiant energy; $100 T=$ percentage transmittance.

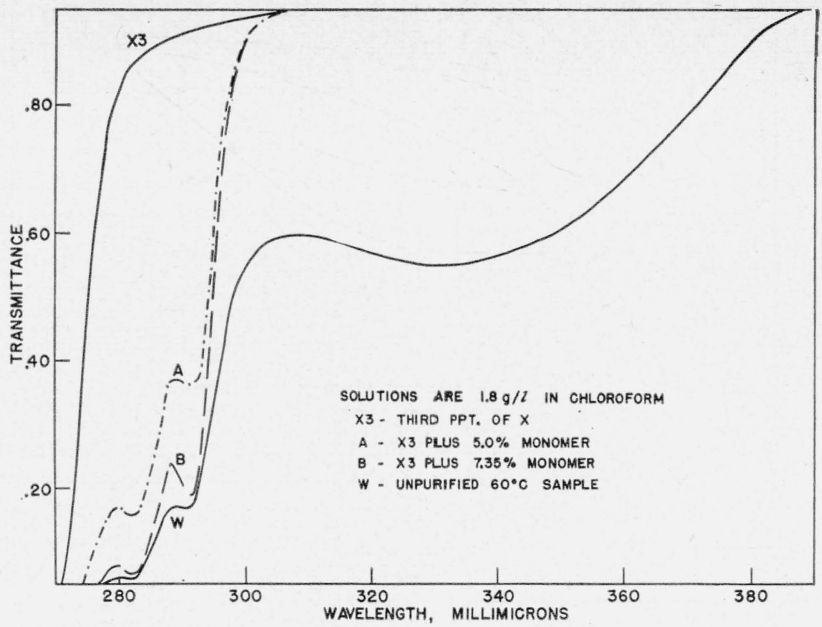

FIGURE 8. Effect of monomer and impurities on the ultraviolet transmittance of.polystyrenes.

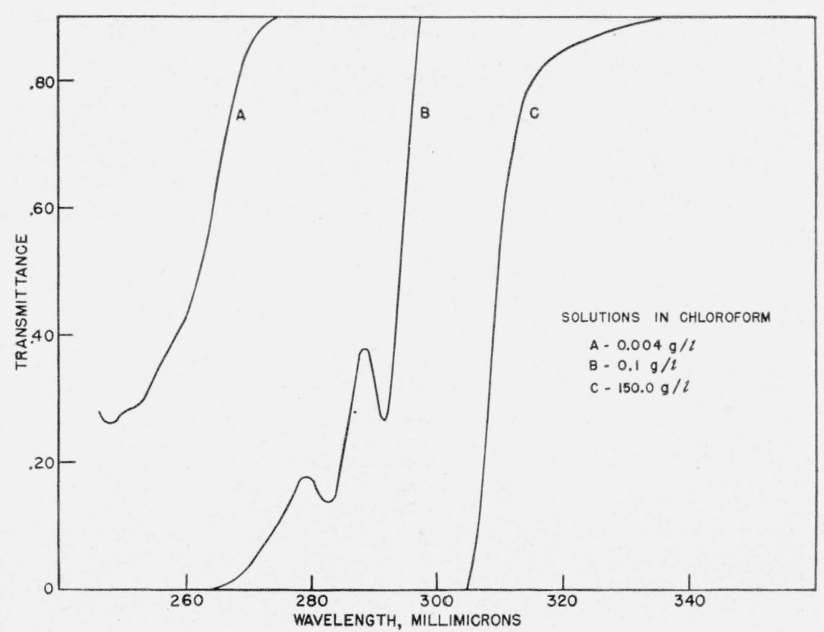

FIGURE 9. Ultraviolet transmittance of styrene monomer.

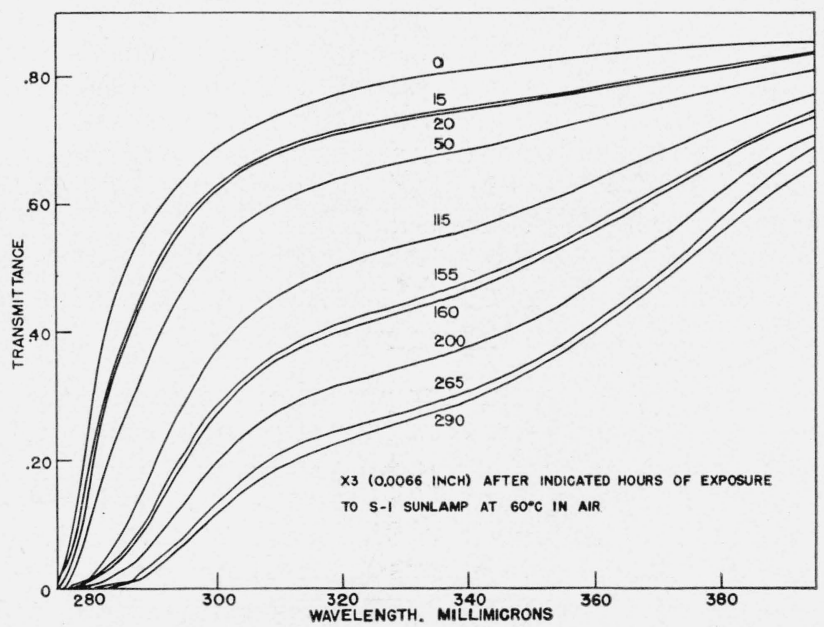

FIgURE 10. Effect of radiant energy on the ultraviolet transmittance of pure polystyrene film. 


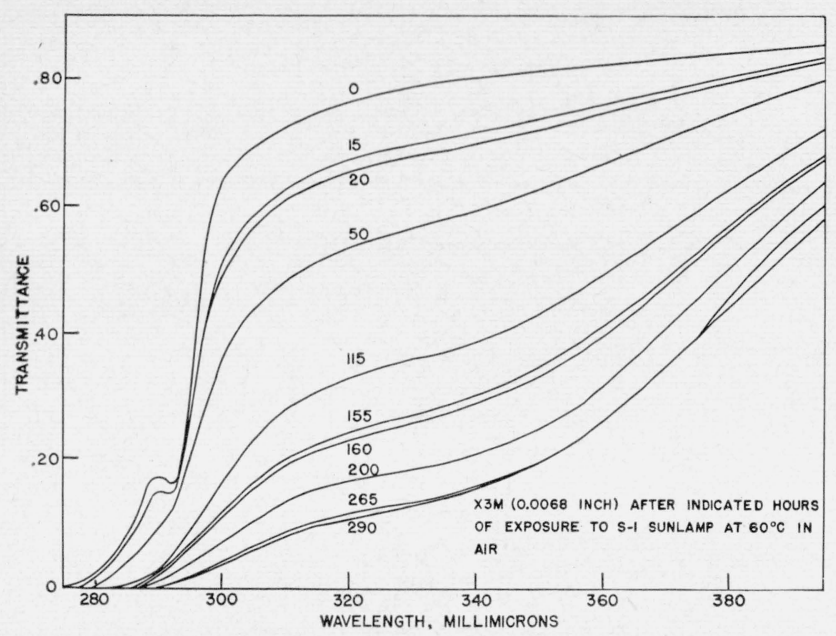

FiguRE 11. Effect of radiant energy on the ultraviolet transmittance of pure polystyrene film plus monomer.

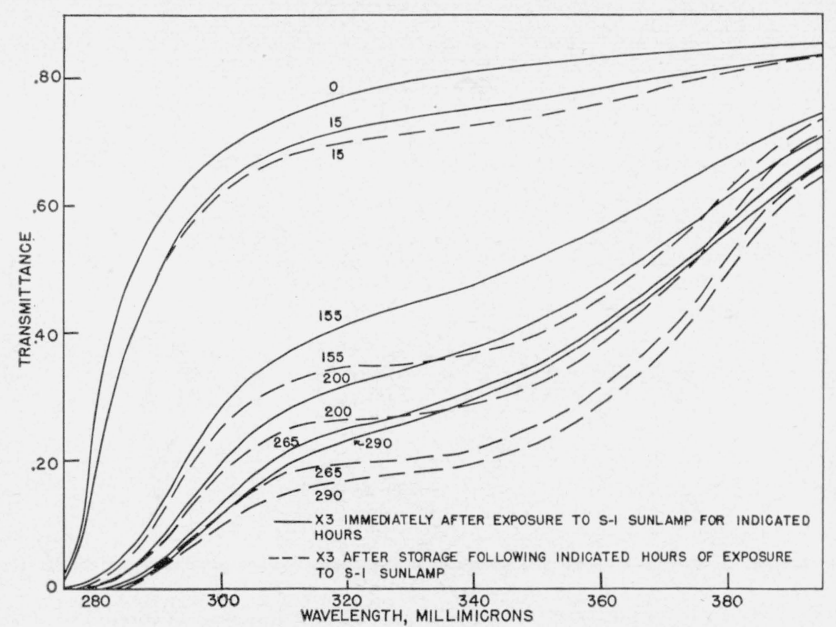

FiguRE 12. Effect of radiant energy and subsequent storage on the ultraviolet transmittance of pure polystyrene film.

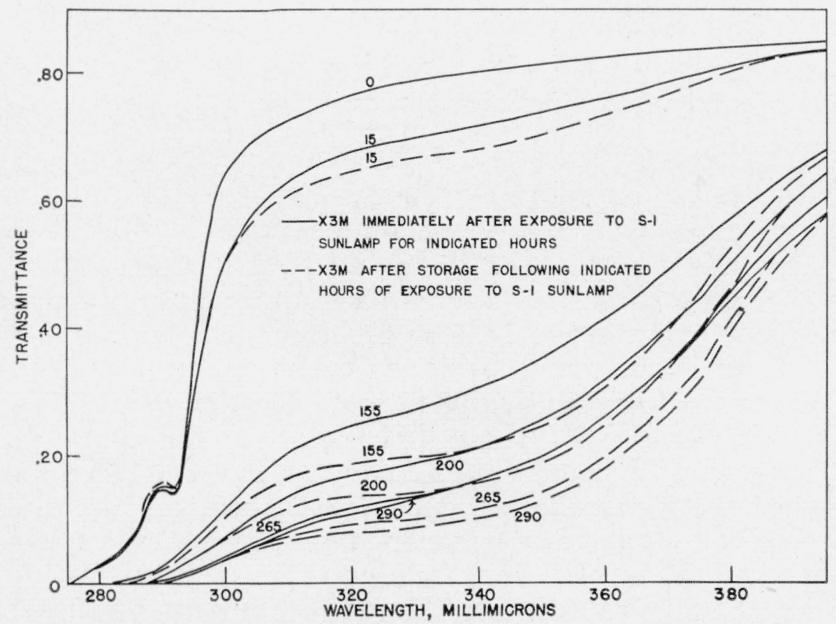

FIGURE 13. Effect of radiant energy and subsequent storage on the ultraviolet transmittance of pure polystyrene film plus monomer.

\section{Post-radiation Changes in Polystyrene Degradation}

In the course of the degradation studies, a postradiation effect was observed. The first indication of this effect was noted when, following the 15-hr S-1 sunlamp exposure, the films were stored in the dark at room conditions in air for $99 \mathrm{hr}$ and their transmittance measured before further exposure. The resulting spectra showed a noticeable increase in absorptance when compared to the spectra measured directly after exposure to radiant energy. The 20- and 25-hr total S-1 exposures followed the pattern of the 15-hr exposure. After $155 \mathrm{hr}$ of total S-1 sunlamp exposure, the films were stored for 13 days, and a very large change in absorptance was apparent when the spectra were measured. The films were then exposed to an additional $5 \mathrm{hr}$ of radiant energy from an $\mathrm{S}-1$ sunlamp, to give a total of $160 \mathrm{hr}$ of exposure, and their spectra remeasured. The increased absorptance apparent after storage was eliminated, and the $160-\mathrm{hr}$ spectra fell in line with the spectra measured immediately upon completion of the $155 \mathrm{hr}$ of exposure. This effect was checked by storing the films for 13-day periods following the 200-, 265-, and 290-hr total exposure times and was found to be real. Figures 12 and 13 present the spectra of the films measured just before and after storage periods. The dashed lines represent the measurements made following the storage periods.

Upon completion of the 290-hr S-1 sunlamp exposure, the spectra of the films were measured at intervals during the ensuing storage of the films in order to determine the length of time necessary for the development of the increased absorptance. When the corrected absorbance, $A_{c},{ }^{5}$ at a particular wavelength is plotted versus the hours of storage following the 290-hr exposure to ultraviolet radiant energy, a curve is obtained for each film which shows that most of the increased absorbance develops in the first $75 \mathrm{hr}$ of storage. Figure 14 is such a plot for numerous wavelengths. From this figure it is seen that the slopes of the curves are very slight at greater than $75 \mathrm{hr}$ of storage and also that a greater and more rapid change occurs at any particular wavelength on storing the monomer-containing film than on storing the pure film.

From this information, it is clear that the first storage-period measurement, which was made after $99 \mathrm{hr}$ of storage following the $15 \mathrm{hr}$ of exposure to ultraviolet radiant energy, is representative of the maximum increase of absorbance due to storage following that S-1 exposure. Two hours of exposure to ultraviolet radiant energy is sufficient to eliminate the absorption increase due to storage. The 202-hr curves and the 267-hr curves were not presented in figures 10 and 11 because they closely follow the 200- and 265-hr curves, with slightly decreased transmittance. One hour of ultraviolet radiant

${ }^{5} A_{c}$ represents the absorbance reading of the film at a given wavelength minus the absorbance reading at that wavelength measured on the film prior to all the absorbance reading at that wavelength measured on the film prior to all
treatment. "Absorbance" is defined as $\log _{10} I 0 / I$; also known as optical density 


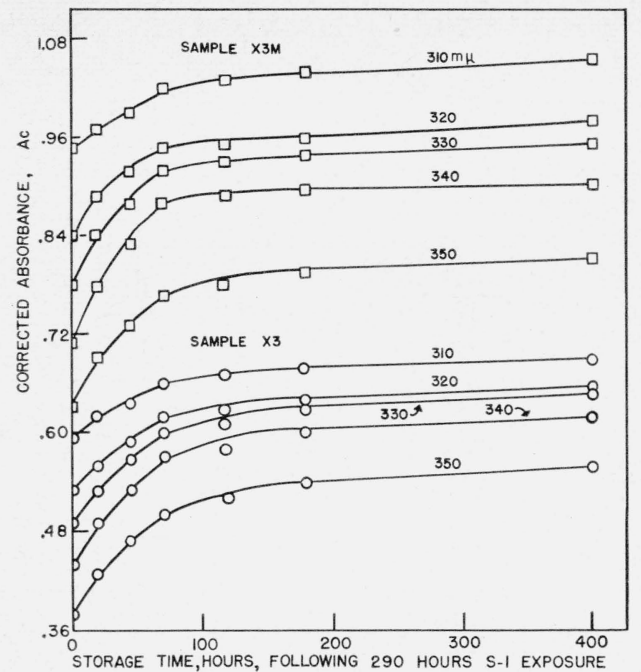

Figure 14. Relation between duration of storage and absorbance of polystyrene films at various wavelengths.

energy, however, was shown to be inadequate to cause this effect, for the 266-hr total-exposure curves fell below 267-hr curves and were of a shape more like that of the storage curves.

Because $2 \mathrm{hr}$ of exposure to ultraviolet radiant energy at $60^{\circ} \mathrm{C}$ in air would return a storage curve to the previous $\mathrm{S}-1$ exposure curve, $2 \mathrm{hr}$ of exposure to $60^{\circ} \mathrm{C}$ heat in air was administered to the films following the storage after the $290-\mathrm{hr}$ total S-1 exposure to observe the effect of heat alone. Heat did not remove the storage absorption band, and there was no change in the transmittance as a result of the treatment.

A graphical presentation of these data, which depicts the complete pattern of treatment of the films, is shown in figure 15. As representative of the absorption band covering the $310-$ to $385-\mathrm{m} \mu$ wavelength region, transmittance at $340 \mathrm{~m} \mu$ is plotted versus exposure time in hours; the storage time (90 hr being considered as maximum storage) is included as well as the time of exposure to $\mathrm{S}-1$ sunlamp radiant energy. This graph reveals the stepwise pattern assumed as the transmittance decreased on exposure to ultraviolet radiant energy, decreased at a different rate on storage, increased sharply on exposure to $2 \mathrm{hr}$ of ultraviolet energy to the value attained preceding storage, and decreased on further exposure to ultraviolet energy, etc.

In order that a study of the rates of increased absorbance of both the pure film and the monomercontaining film for both the exposures to ultraviolet energy and the storage treatments following them could be made, plots of the corrected absorbance, $A_{c}$, at various wavelengths versus hours of exposure to ultraviolet radiant energy were made. Figure 16 is such a plot for $340-\mathrm{m} \mu$ wavelength. The dashed lines are drawn through the readings made after 13 days of storage following the particular exposure to ultraviolet energy. The plots for the pure film are

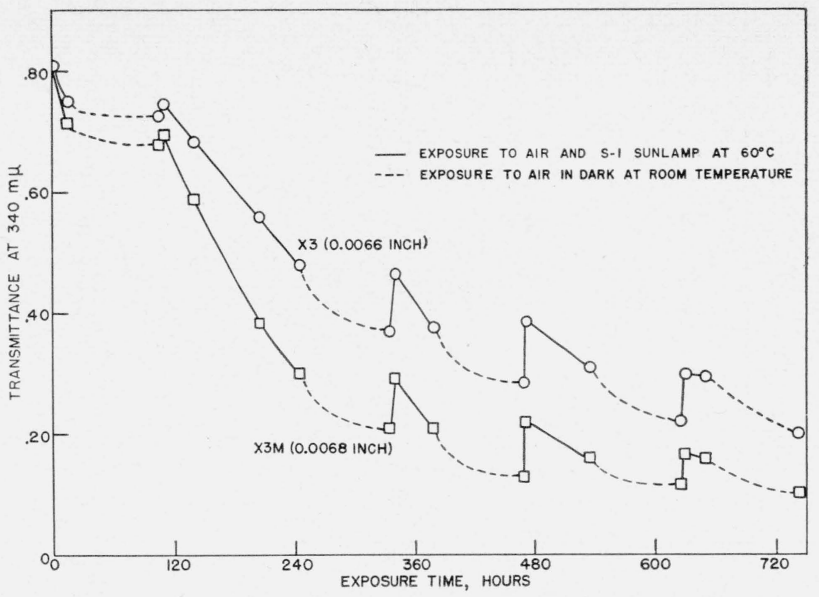

Figure 15. Effect of exposure to radiant energy and to subsequent storage on the ultraviolet transmittance of polystyrene film at $340 \mathrm{m \mu}$.

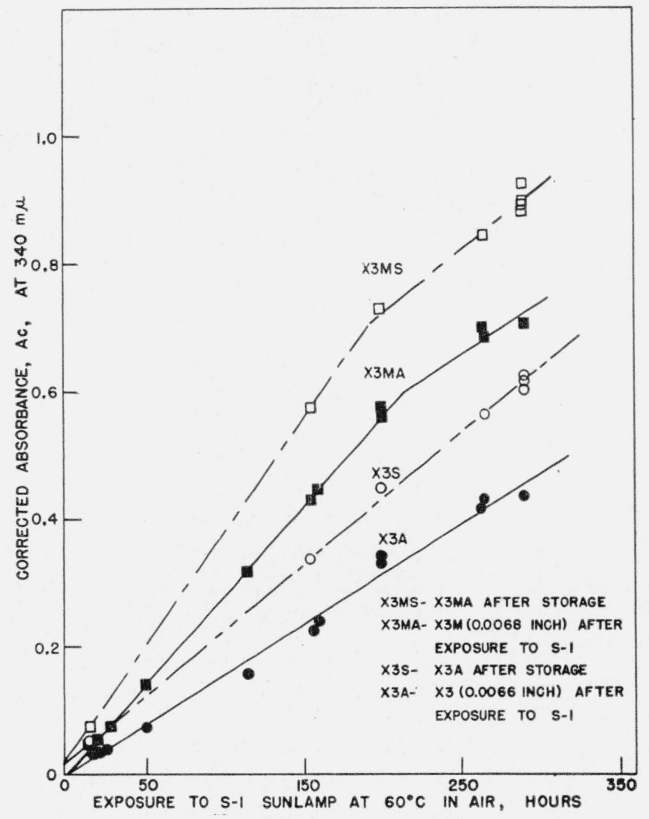

FiguRE 16. Relations between exposures and absorbance of polystyrene films at $3.40 \mathrm{m \mu}$.

linear but the plots for the monomer-containing film appear to deviate from linear behavior following the 200-hr exposure. With up to 200-hr treatment, the plots for the monomer-containing film have steeper slopes than those for the pure film, but at longer exposures the slopes seem to be equal, the curves being practically parallel to each other.

The slopes of these lines plotted for numerous wavelengths were calculated and gave the rates of increased absorbance with treatments of the films for the various wavelengths. These rates are presented in the first four columns of table 2. In an attempt to estimate the rate of increase of absorbance due to storage alone, the rate due to the exposure to ultraviolet energy was subtracted from the 


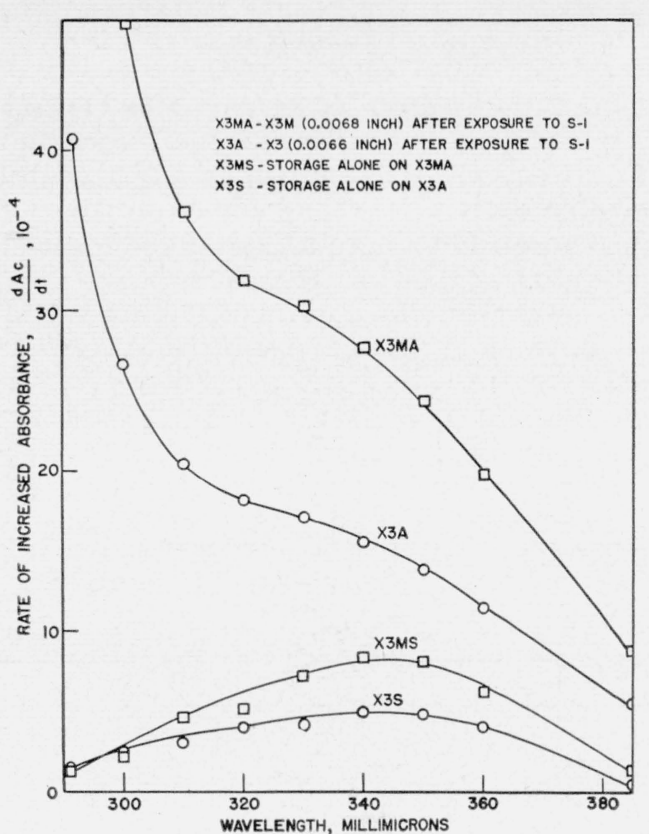

FIGURE 17. Effect of exposures on absorbance of polystyrene films.

rate calculated from the measurements made following the storage periods. Columns 5 and 6 of table 2 list these rates of increased absorbance due to storage alone for the various wavelengths.

If the rates of increased absorbance listed in columns $1,2,5$, and 6 of table 2 are plotted versus wavelength of absorbance (fig. 17), the difference between the absorption curves obtained from films exposed to ultraviolet energy and films subsequently stored in the dark is emphasized. The greatest rate of change in the absorption curves obtained from exposure to ultraviolet energy alone is seen to be at the shorter wavelengths. The maxima in the two lower curves indicate that $340 \mathrm{~m} \mu$ is the wavelength of greatest rate of change of absorbance on storage of the films.

TABLE 2. Rates of increased absorbance, $d . A_{c} / d t$

Values for pure polysytrene film are based on exposures to radiant energy irom an S-1 sunlamp up to 290 hours, Values for pure polystyrene film plus monomer are based on exposures up to 200 hours.

\begin{tabular}{|c|c|c|c|c|c|c|}
\hline \multirow{3}{*}{$\begin{array}{l}\text { Wave- } \\
\text { length } \\
\text { plotted }\end{array}$} & \multicolumn{2}{|c|}{$\begin{array}{l}\text { Exposure of film to } \\
\mathrm{S}-1 \text { sunlamp at } \\
60^{\circ} \mathrm{C} \text { in air }\end{array}$} & \multicolumn{2}{|c|}{$\begin{array}{l}\text { Exposure of film to } \\
\mathrm{S}-1 \text { sunlamp plus } \\
\text { storage in dark }\end{array}$} & \multicolumn{2}{|c|}{$\begin{array}{l}\text { Storage of film in } \\
\text { dark }\end{array}$} \\
\hline & Pure & $\begin{array}{c}\text { Plus } \\
\text { monomer }\end{array}$ & Pure & $\begin{array}{c}\text { Plus } \\
\text { monomer }\end{array}$ & Pure & $\begin{array}{c}\text { Plus } \\
\text { monomer }\end{array}$ \\
\hline & 1 & 2 & 3 & 4 & 5 & 6 \\
\hline $\begin{array}{c}m_{\mu} \\
300 \\
310 \\
320 \\
330 \ldots \\
340 \\
350 \\
360 \\
385\end{array}$ & $\begin{array}{r}0.0027 \\
.0020 \\
.0018 \\
.0017 \\
.0015 \\
.0014 \\
.0012 \\
.0005\end{array}$ & $\begin{array}{r}0.0048 \\
.0036 \\
.0032 \\
.0030 \\
.0028 \\
.0024 \\
.0020 \\
.0009\end{array}$ & $\begin{array}{r}0.0029 \\
.0023 \\
.0022 \\
.0021 \\
.0020 \\
.0019 \\
.0016 \\
.0005\end{array}$ & $\begin{array}{r}0.0050 \\
.0041 \\
.0037 \\
.0037 \\
.0036 \\
.0032 \\
.0026 \\
.0010\end{array}$ & $\begin{array}{r}0.0002 \\
.0003 \\
.0004 \\
.0004 \\
.0005 \\
.0005 \\
.0004 \\
.0000\end{array}$ & $\begin{array}{r}0.0002 \\
.0005 \\
.0005 \\
.0007 \\
.0008 \\
.0008 \\
.0006 \\
.0001\end{array}$ \\
\hline
\end{tabular}

\section{Sources of Characteristic Absorption Bands in a Degraded Polystyrene}

\subsection{Benzaldehyde}

It is apparent that exposure to ultraviolet radiant energy leads to the alteration of the polymer so that it absorbs in a characteristic manner, and that subsequent storage leads to further alteration which causes absorptance in a different characteristic manner. It was also noted that the absorption band that developed on storage was similar to the "impurity" band observed in the impure polymers. This similarity is observed by comparing the curve of impure sample $\mathrm{X}$ in figure 6 with the curve of pure polymer exposed to $155 \mathrm{hr} \mathrm{S}-1$ plus storage shown in figure 12 .

Because infrared spectrophotometric studies of polystyrene films indicated the presence of carbonyl and hydroxyl groups after treatment with ultraviolet radiant energy, and previous mass spectrometric measurements of gaseous products formed on degradation [3] showed some formaldehyde and benzaldehyde, benzaldehyde ${ }^{6}$ was examined in the ultraviolet region. It gave the curves shown in figure 18 for $0.1-, 1.0-$, and $2.0-\mathrm{g}$ /liter solutions in chloroform. On addition of 1.6 percent of benzaldehyde to an 18-g/liter solution of pure polymer X3 in chloroform, the spectrum obtained (fig. 19) showed a resemblance in shape to the observed curves obtained on storage after exposure of the polystyrene films to ultraviolet energy. However,

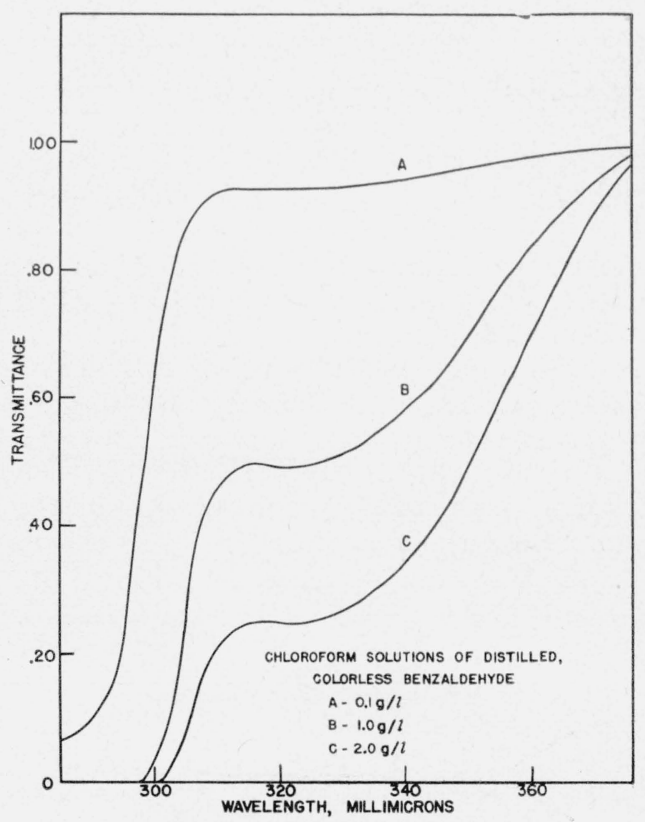

FiguRE 18. Ultraviolet transmittance of benzaldehyde.

6 The benzaldehyde was not purified but contained oxidation products, and was, therefore, considered to be more representative of the material possibly present in the polymer. 


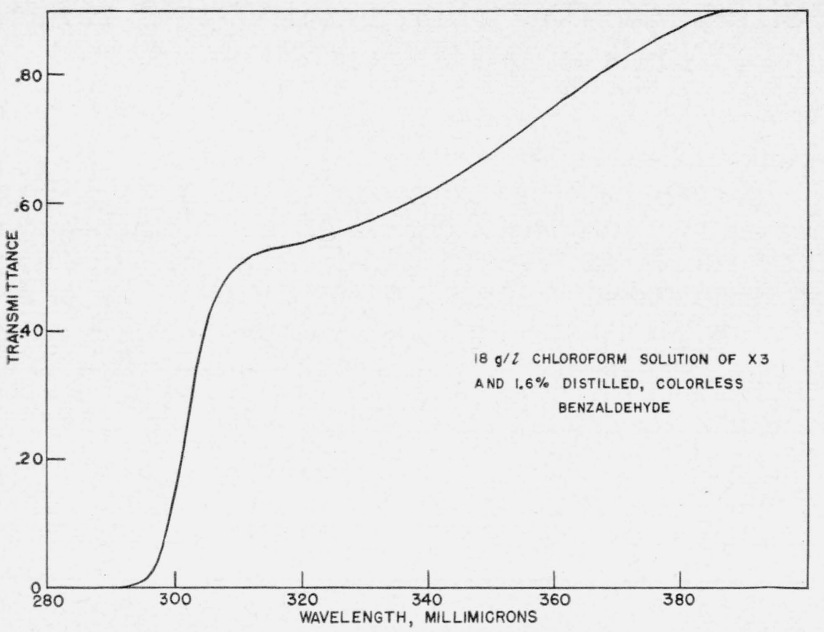

FIGURE 19. Ultraviolet transmittance of polystyrene contaminated with benzaldehyde.

the absorptance shown in figure 19 is not as broad and not as intense at $340 \mathrm{~m} \mu$ as that which develops on storage. Also, there is an intense absorptance below $300 \mathrm{~m} \mu$; storage causes little change at these shorter wavelengths (fig. 17). The $K_{291.5} *$ of benzaldehyde is 9.67 , whereas its $K_{320}$ is 0.3 ; therefore, the presence of an unreasonably high concentration. of benzaldehyde in the sample would be required to give the long wavelength absorption. Hence, it is not plausible to assume that the absorption pattern caused by exposure to ultraviolet energy can be attributed to the presence of benzaldehyde alone.

\subsection{Conjugated Carbonyl-Containing Compounds}

Other compounds containing a more extended system of conjugation including the carbonyl group were therefore investigated in order that one having an extinction coefficient sufficient to explain either type of absorption might be found. The APIRP collection of spectra [11] and the Friedel and Orchin collection [12] show numerous compounds which exhibit absorbance in these regions. Those compounds exhibiting absorption bands similar in shape to those under discussion usually contain one or the other of the following structures:

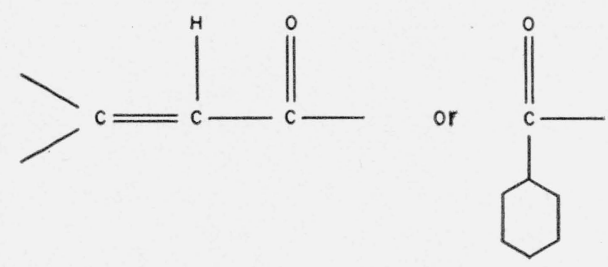

${ }^{*} K=$ specific extinction coefficient at indicated wavelength in millimicrons= absorbance for a concentration of $1 \mathrm{~g} / \mathrm{liter}$.
Some examples are: benzoin, benzophenone, mesityl oxide, methyl vinyl ketone, crotonaldehyde, benzoyl acetone, and $p$-toluyl acetone. However, the extinction coefficients at the desired wavelengths are again quite small. The latter two compounds have appreciable extinction coefficients, but the maxima of the absorption bands are shifted to 310 and $317 \mathrm{~m} \mu$, respectively.

While benzaldehyde and its oxidation products [13] do not have high enough extinction coefficients to account for the observed absorbance in the polystyrene films, it is likely that related structures such as those shown below might be present in the system, and their oxidation products might easily have sufficient conjugation to give very large extinction coefficients:
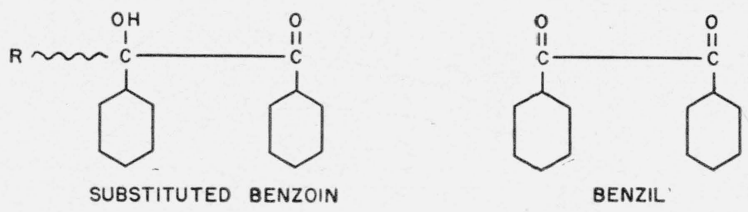

There is also the possibility of a reaction similar to an aldol condensation, once carbonyl is formed:

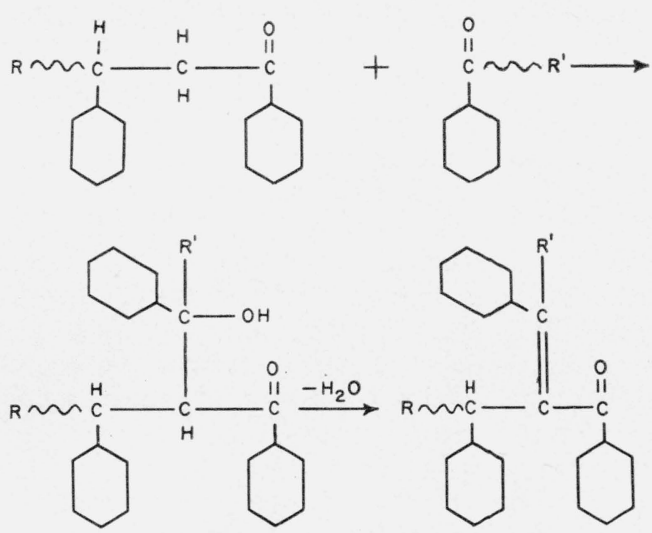

This condensation reaction results in a final product with a high degree of conjugation. Both pure and monomer-containing films may undergo this type of reaction on exposure to ultraviolet radiant energy, once the carbonyl is formed. Monomer styrene has a reactive site for oxidation and reacts to form carbonyl compounds of lower molecular weight, such as formaldehyde and benzaldehyde, thus affording greater mobility for reaction than might be expected from larger carbonyl-containing compounds.

The yellow color evidenced by partially oxidized benzaldehyde is caused by large amounts of a material of a high degree of conjugation in the oxidation products. Yellowing of polystyrene films can be explained in a similar manner if structures like the above are postulated. Infrared studies confirm that carbonyl structures do exist in polystyrene after exposure to ultraviolet radiant energy in air as mentioned previously. 


\subsection{Stable Free Radical Compounds}

It is possible that the absorbance of the film ${ }^{-i m}$ mediately following exposure to ultraviolet radiant energy is the absorbance of a relatively stable free radical whose structure can shift to a higher order of conjugation on storage in the dark, only to be reactivated by ultraviolet radiant energy at a later time. Such possible mechanisms might be of the types:
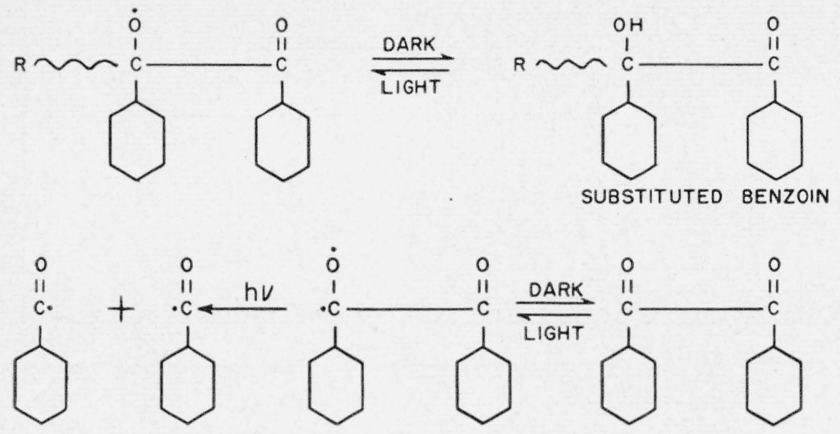

BENZIL

Both compounds would be relatively nonvolatile at the temperatures of the exposure and storage conditions used in this work. Infrared spectra of the films in a post-radiated state were nearly identical with spectra of the films made immediately following an exposure period. Therefore, no change in carbonyl or hydroxyl content between the two states could be so detected; but such a change might be too small to be measurable by this method.

The reaction occurring in the dark is not continuously progressive as is the reaction in ultraviolet radiant energy in the sense that most of the storage degradation products after any one exposure are formed in the first $75 \mathrm{hr}$ of storage and very little more is formed even after as many as 64 days of storage. The reaction in the dark does not seem to affect the rate of degradation by ultraviolet radiant energy, but the reaction in ultraviolet governs the reaction in the dark. In the first place, the reaction in the dark requires a previous $\mathrm{S}-1$ sunlamp exposure for it to occur. Specimens of the pure film and of the monomer-containing film were stored for 4 months in the dark at room temperature with no other treatment, and their ultraviolet transmittance showed no change during that time. Second, the amount of storage product formed at any one time is proportional to the total number of hours of exposure to ultraviolet radiant energy received at that time. Even though an exposure to ultraviolet energy immediately following a storage period seems to counteract the effect of storage, the following storage period shows an increase in the development of storage product over the previous period due to the additional exposure to ultraviolet radiant energy. These facts seem to support the assumption of the formation on exposure to ultraviolet radiant energy of relatively stable free radicals which shift to structures of more conjugation on storage; the latter are, in turn, reactivated and more free radicals formed by further exposure to ultraviolet radiant energy.

These "stable" free radicals probably can exist because the polymer is exposed and measured in the solid state. Evidence for the presence of long-lived free radicals in materials of rigid or crystalline structure is increasing day by day $[14] .^{7}$ The lack of mobility of polymer chains in a material which is held below its glass-transition temperature ${ }^{8}$ is the most logical explanation for entrapment of free radicals on the end of polymer chains. Termination reactions of such radicals are extremely slow due to such low mobility, and the slow diffusion of oxygen and other gaseous materials into such a mass would also severely limit the rate of reaction of these materials with the active parts of the polymer in the interior of the sample.

The presence of styrene monomer in the polymer apparently augments the degradation of the polymer chain, as well as producing monomer oxidation products, such as formaldehyde and benzaldehyde through free radical reactions $[15,16]$. In the case studied here, the addition of styrene monomer to the polymer has resulted in degradation of the sample, as followed by ultraviolet transmission measurements, at a rate almost double that of the pure polymer (see table 2 and fig. 17). Infrared measurements made on the films after the total exposure of $290 \mathrm{hr}$ to ultraviolet radiant energy from an S-1 sunlamp showed a very appreciable difference between the two films; the carbonyl and hydroxyl absorption bands of the monomer-containing film are almost double those of the pure film. Figure 20 illustrates these differences as well as the other spectral changes which occurred. The carbonyl group absorptance is shown in the region of 5.7 to $6.0 \mu$ and that of the hydroxyl in the region of $2.85 \mu$. The spectrum of each film after $290 \mathrm{hr}$ of S-1 sunlamp radiation is shown in this figure along with a spectrum of an untreated film which is common to both. This comparison is possible because the monomer that was added and remained in the one film was insufficient to cause any change in the infrared spectrum of the untreated polymer, although sufficient to effect an appreciable change in the degradation. The increase in degradation caused by the addition of monomer is not noticeable in the rates computed from the ultraviolet transmission measurements after approximately $200 \mathrm{hr}$ of exposure to S-1 sunlamp radiant energy. This change in rate indicates that the added monomer had probably completely reacted by that time and thence the degradation of this film proceeded as in the pure film.

\footnotetext{
${ }^{7}$ Such evidence was summarized by William O. Baker of the Bell Telephone Laboratories, Murray Hill, N. J., in a lecture at the Polytechnic Institute of Brooklyn on January 17, 1953, entitled "Entrapped Polymer Radicals." Brooklyn on January 17, 1953, entitled "Entrapped Poly
8 Sometimes called second-order transition temperature.
} 


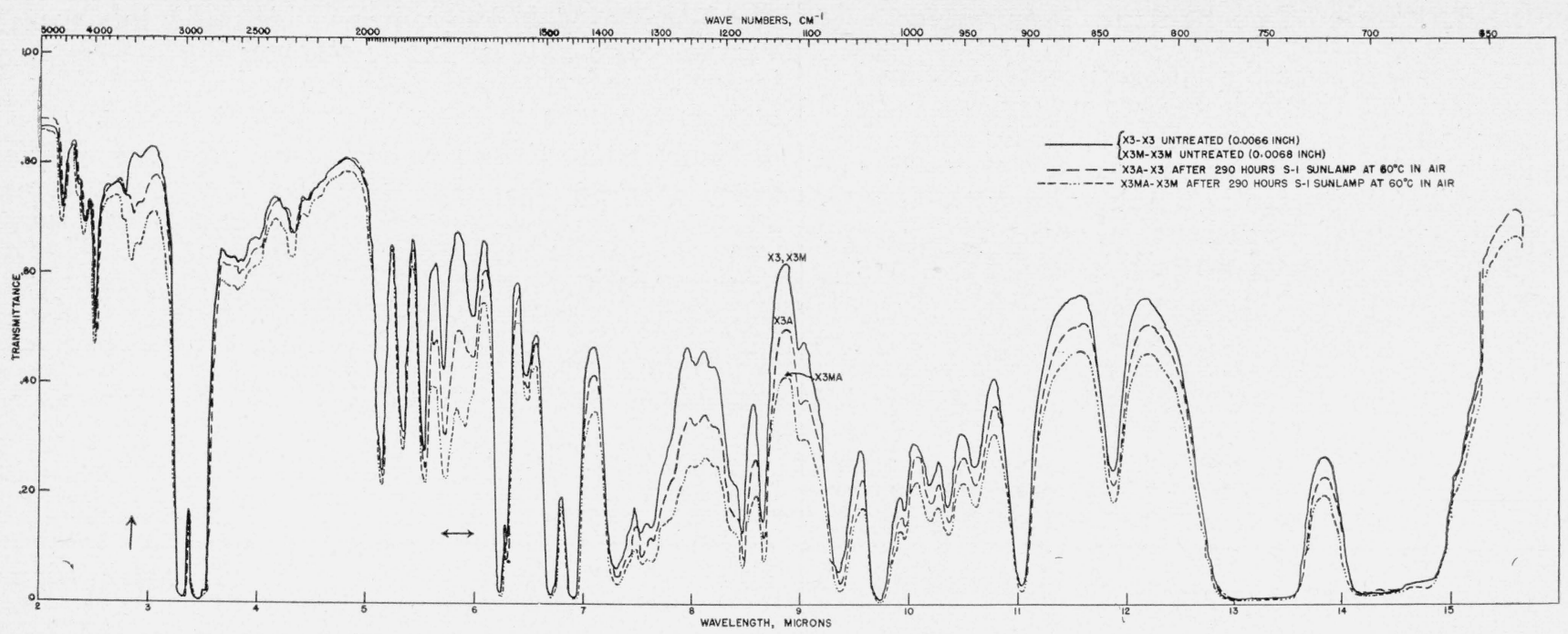

FICURE 20. Effect of exposure to radiant energy on the infrared transmittance of polystyrene films.

\subsection{Oxidation Products}

The mechanisms that have been postulated for the degradation of polystyrene in ultraviolet radiant energy, however, are probably not all the possible reactions because polystyrene most likely is not a linear hydrocarbon polymer. It has been shown to contain oxygen in the chain [17]. Portions of catalysts, when used, are also part of the chain. All these factors offer weak links for the initiation of degradation and also can alter the products formed on exposure to ultraviolet radiant energy, depending on their type and number. The presence of a multicomponent system is borne out in part by the fact that the absorption curve in the region 290 to 400 $\mathrm{m} \mu$ for the exposed samples does not exhibit a sharp absorption peak, but rather a broad band, indicating a mixture of compounds absorbing in this general region.

Boyer [18] has described many of the factors affecting the ultraviolet absorbance of a degraded halogen-containing copolymer. Those factors applicable to the explanation of the absorbances noted in the present case of degraded polystyrene include an inductive effect of the conjugated systems on each other, the influence of the presence of a mixture of isomers, the coexistence of many degrees of conjugation, and the positional isomerization of double bonds. However, because of the presence of the phenyl groups and the oxidized structures in polystyrene, a long linear conjugated system is not required to produce the absorbance noted above $300 \mathrm{~m} \mu$ as is necessary in aliphatic polymers in the absence of air. Boyer also states that in the case of exposure to light, it will be those regions of the chain that have already developed unsaturation and conjugation that will absorb radiant energy most strongly and over a progressively widening spectral band. Although the oxidation and production of unsaturation may be a random process ini- tially, the localization of absorbed energy probably then promotes unsaturation in adjacent monomer units leading to the formation of further conjugated systems.

The most reasonable approach to understanding the steps of the reaction in ultraviolet radiant energy would be to study more simple parent compounds than polystyrene. Styrene monomer, cumene, and benzaldehyde appear to be the most likely compounds for initial study of oxidation mechanisms promoted by ultraviolet radiant energy. Investigations of the reactions occurring in the dark following exposure to radiant energy should be studied with pure materials of definitely known structure which are solids at the temperatures used. Such compounds are typified by $n$-dotriacontane and a similar branched saturated hydrocarbon. Materials whose glass-transition temperatures are above room temperature would be desirable at the start. This factor would increase the possibility of obtaining entrapped free radicals in the mass of the material.

\section{Effect of Thermal Energy on Transmittance}

The initiation of degradation in these experiments was apparently induced by the ultraviolet radiant energy and not by the approximately $60^{\circ} \mathrm{C}$ temperature at which the exposures were conducted. It has been pointed out previously that $2 \mathrm{hr}$ of heat at $60^{\circ} \mathrm{C}$ in air following a storage period in which absorption occurred will not eliminate any of the absorption band formed during storage. Heating at this temperature in the absence of ultraviolet radiant energy will not cause degradation of the polystyrene such as that caused by exposure to ultraviolet radiant energy at $60^{\circ} \mathrm{C}$.

Specimens of the pure film and of the monomercontaining film which had been stored for 4 months 
in the dark at room temperature were heated in an air oven at 60 to $63^{\circ} \mathrm{C}$ for a total of $290 \mathrm{hr}$. Ultraviolet transmission measurements after intermittent heat and storage periods showed no long wavelength absorption band and almost no change at all in the over-all spectrum. The films also did not exhibit any discoloration. Previous infrared measurements on films subjected to $100^{\circ} \mathrm{C}$ for $200 \mathrm{hr}$ [2] did not show any production of earbonyl or hydroxyl absorption bands or any other spectral change.

\section{Summary and Conclusions}

Pure polystyrene film exposed to $290 \mathrm{hr}$ of ultraviolet radiant energy from an $\mathrm{S}-1$ sunlamp at $60^{\circ} \mathrm{C}$ in air was degraded as shown by decreased ultraviolet transmittance. The absorbance increased linearly with exposure time and the greatest rate of increase was calculated to be at wavelengths shorter than $300 \mathrm{~m} \mu$.

Storage of pure polystyrene film in the dark in air at room temperature following exposure to an S-1 sunlamp caused the development of a definite broad absorption band with a maximum at $340 \mathrm{~m} \mu$. Subsequent exposure to ultraviolet radiant energy caused the elimination of the absorption band developed on storage.

The formation of the post-radiation products was governed by the S-1 radiant energy; storage without previous ultraviolet exposure caused no change in the ultraviolet spectrum. The formation of the storage products, however, did not alter the rate of degradation by ultraviolet radiant energy.

Exposure of the polymer film to heat at $60^{\circ} \mathrm{C}$ in air for $290 \mathrm{hr}$ with intermittent storage caused no change in the ultraviolet spectrum, the degradation shown by films exposed to S-1 radiant energy at this temperature being induced by the $\mathrm{S}-1$ radiant energy and not by the accompanying heat.

The addition of monomer to pure polymer did not change the pattern of degradation, as shown by ultraviolet spectrophotometry, but did cause the over-all degradation to occur at an increased rate; the accelerated rate held until all the monomer was apparently spent and thence the reaction proceeded as for pure polymer.

Reactions of the type that may account for the absorption curves noted on exposure to ultraviolet radiant energy and on subsequent storage are postulated.

The shape of the ultraviolet absorption curves of the purified polymer after exposure to ultraviolet radiant energy and subsequent storage closely resembles the shape of the initial spectra obtained on commercial polystyrene samples, which indicates that the impurities in the commercial products are of the same chemical types as those produced on exposure of pure polymer to ultraviolet radiant energy and subsequent storage. In most cases the commercial samples absorbed more strongly than the degraded purified polymer.
The authors express their appreciation to Shigeru Ishihara for valuable suggestions during the conduct of the work and to C. Brown and H. A. Baker for preparation of the figures.

This investigation was sponsored by, and conducted with financial assistance from the Office of the Quartermaster General, Department of the Army.

\section{References}

[1] L. A. Matheson and R. F. Boyer, Light stability of polystyrene and polyvinylidene chloride, Ind. Eng. Chem, 44, 867-74 (1952).

[2] B. G. Achhammer, M. J. Reiney, and F. W. Reinhart, Study of degradation of polystyrene, using infrared spectrophotometry, J. Research NBS 47, 116 (1951) RP2235.

[3] B. G. Achhammer, M. J. Reiney, L. A. Wall, and F. W. Reinhart, Study of degradation of polystyrene by means of mass spectrometry, J. Polymer Sei. VIII, No. 5, 555 (1952).

[4] Plastics, organic: general specifications, test methods. Federal Specification L-P-406a (Government Printing Office, Washington 25, D. C., Jan. 24, 1944).

[5] A. Smakula, Zur Bestimmung der Molekulargewichte von Polystyrolen, Angew. Chem. 4\%, 777 (1934).

[6] J. J. McGovern, J. M. Grim, and W. C. Teach, Determination of monomer in polystyrene. Spectrophotometric and solubility methods, Anal. Chem. 20, 312 (1948).

[7] E. J. Meehan, Spectrophotometric determination of the styrene content of butadiene-styrene copolymers, J. Polymer Sci. I, 175 (1946); Rubber Chem. and Tech. 19, 1077 (1946).

[8] J. E. Newell, Residual monomer in polystyrene. Spectrophotometric analysis, Anal. Chem. 23, 445 (1951).

[9] T. W. Campbell, S. Linden, S. Godshalk, and W. G. Young, The absorption spectra of some benzene derivatives with unsaturated side chains, J. Am. Chem. Soc. 69, 880 (1947).

[10] N. Hadden and J. A. Perry, Ultraviolet spectrophotometric determination of styrene in ethylbenzene. Anal. Chem. 23, 1337 (1951).

[11] Catalog of ultraviolet absorption spectrograms, issued by the American Petroleum Institute Research, Project 44 at the NBS.

[12] R. A. Friedel and M. Orchin, Ultraviolet spectra of aromatic compounds (John Wiley \& Sons, Ine., New York, N. Y., and Chapman \& Hall, Ltd., London, 1951).

[13] H. L. J. Backstrom, Der Kettenmechanismus bei der Autoxydation von Aldehyden, Z. physik. Chem. B25, 99-121 (1934).

[14] L. A. Wall and M. Magat, Dégradation des polymères par les rayons $\gamma$ et les neutrons, J. chim. phys. 50, 308 (1953); also, Effects of atomic radiation on polymers, Modern Plasties 30, No. 11, 111 (1953).

[15] S. Medvedev and P. Zeitlin, Oxidation and polymerization of styrene, Acta Physicochim. URSS 20, 3 (1945).

[16] H. Boardman and P. W. Selwood, The oxidation of styrene, J. Am. Chem. Soc. 72, 1372 (1950).

[17] F. A. Bovey and I. M. Kolthoff, The mechanism of emulsion polymerization III. Oxgyen as a comonomer in the emulsion polymerization of styrene, J. Am. Chem. Soc. 69, 2143 (1947).

[18] R. F. Boyer, A statistical theory of discoloration for halogen-containing polymers and copolymers, J. Phys, and Colloid Chem.51, 80 (1947).

Washington, May 22, 1953. 